01.1;08.3;09.4

\title{
Гибридный металлополимер как потенциальная активная среда оптоакустического генератора
}

\author{
(C) Е.И. Гиршова ${ }^{1,2}$, Е.П. Микитчук ${ }^{3}$, А.В. Белоновский ${ }^{1,2}$, К.М. Морозов ${ }^{1,2}$ \\ ${ }^{1}$ Санкт-Петербургский национальный исследовательский Академический университет им. Ж.И. Алфёрова РАН, \\ Санкт-Петербург, Россия \\ ${ }^{2}$ Университет ИТМО, Санкт-Петербург, Россия \\ 3 Белорусский государственный университет, Минск, Беларусь \\ E-mail: ilinishna@gmail.com
}

Поступило в Редакцию 5 июля 2021 г.

В окончательной редакции 11 октября 2021 г.

Принято к публикации 23 октября 2021 г.

Исследован гибридный материал, состоящий из полидиметилсилоксана и распределенных по всему его объему наночастиц серебра, рассчитаны его оптические и термодинамические характеристики для разных объемных долей содержания серебра. Теоретически показано, что данный материал при объемной доле серебра около 30\% может быть использован в качестве активной среды оптоакустического преобразователя с рабочим диапазоном частот порядка $10 \mathrm{MHz}$.

Ключевые слова: гибридный материал, ультразвуковой генератор, таммовский плазмон, полидиметилсилоксан.

DOI: 10.21883/PJTF.2022.03.51981.18948

Принцип работы оптоакустического генератора основан на расширении и сжатии активной области прибора за счет нагрева вследствие поглощения лазерных импульсов и последующей релаксации $[1,2]$. Исследования оптоакустических методов в нашей стране систематически проводились с середины 70-х годов прошлого века группой под руководством Л.М. Лямшева и К.А. Наугольных в Акустическом институте им. акад. Н.Н. Андреева и другими коллективами $[3,4]$. Для максимальной эффективности преобразования энергии лазерного излучения в механическую необходимо, чтобы материалы, входящие в состав прибора, имели правильно подобранные оптические, термодинамические и механические характеристики. Во-первых, должно быть реализовано полное поглощение лазерного излучения на длине волны лазера; во-вторых, материал должен иметь высокий коэффициент теплового расширения; в-третьих, активная область должна остывать достаточно быстро, чтобы система успевала релаксировать перед поглощением следующего импульса [5].

Поскольку материал активной среды должен удовлетворять одновременно нескольким требованиям, найти одно универсальное вещество крайне трудно. Но можно создать гибридный материал, который будет совмещать полезные свойства его компонентов и нивелировать их недостатки. В данный момент гибридные материалы активно применяются в фотонике, в частности, в качестве светоизлучающих материалов [6,7], в конструкциях солнечных панелей [8], для управления терагерцевым излучением [8-10].

В качестве материала для активной среды оптоакустических генераторов часто выбирают полидиметил- силоксан, так как он обладает феноменально высоким коэффициентом температурного расширения $[11,12]$. Однако сам по себе он не способен эффективно поглощать лазерное излучение, поэтому должен использоваться слой металла [13] или металлические наночастицы [14]. Успешная реализация подобных схем оптоакустических генераторов уже описана в литературе [15]. Главным техническим недостатком таких конструкций является ограничение, накладываемое на рабочую длину волны: для хорошего поглощения в металле нужно использовать лазеры сине-зеленого диапазона. Было показано [5], что использование структур с таммовским плазмоном позволяет добиваться полного поглощения на длине волны полупроводниковых лазеров на основе GaAs, использование которых является предпочтительным ввиду их доступности, высокой мощности и широких возможностей модуляции излучения.

Для моделирования параметров гибридного материала, который может быть использован в качестве активной среды оптоакустического генератора, был выбран полидиметилсилоксан с интегрированными наночастицами серебра. Полидиметилсилоксан является теплоизолятором, поэтому в сплошном слое перераспределение температуры идет очень медленно. Можно оценить характерное время выравнивания температуры в слое вещества по формуле [5]:

$$
\tau=\frac{d^{2}}{2 D},
$$

где $d$ - толщина слоя, $D-$ температуропроводность полидиметилсилоксана. В таком случае получается, что характерное время распределения температуры по слою толщиной $500 \mathrm{~nm}$ составляет порядка $1000 \mathrm{ns,} \mathrm{что} \mathrm{не}$ 

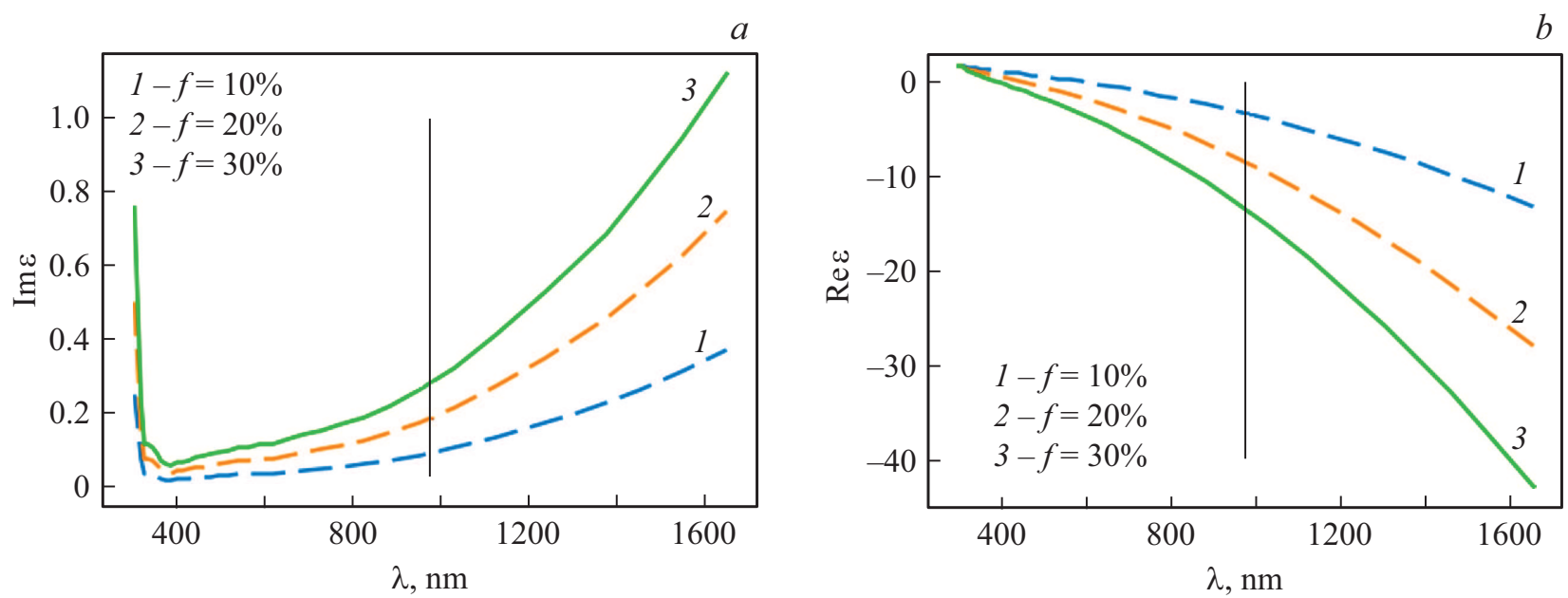

Рис. 1. Спектры мнимой $(a)$ и вещественной $(b)$ частей эффективной диэлектрической проницаемости гибридного материала для различных объемных долей серебряных наночастиц. Вертикальной линией отмечена рабочая частота полупроводниковых лазеров на основе GaAs $(980 \mathrm{~nm})$.

позволяет рассчитывать на адекватное функционирование при частотах выше $1 \mathrm{MHz}$, когда как в медицине и дефектоскопии востребованы ультразвуковые волны в диапазоне 2-29 MHz. Для решения этой проблемы был выбран гибридный материал, позволяющий прогревать органический слой равномерно по всему объему.

Для того чтобы разработать дизайн структуры с таммовским плазмоном на базе гибридного материала, необходимо знать его эффективные оптические характеристики, такие как показатели преломления и поглощения. Для двухкомпонентной гетерогенной системы, в которой объемная доля одного из компонентов не превышает одной трети, может быть применена формула Максвелла-Гарнетта, выражающая диэлектрическую проницаемость смеси $\varepsilon_{m i x}$ через объемную долю включений $(f)$ и диэлектрические проницаемости компонентов:

$$
\varepsilon_{\text {mix }}=\varepsilon_{\mathrm{PDMS}}\left[1-\frac{3 f\left(\varepsilon_{\mathrm{PDMS}}-\varepsilon_{\mathrm{Ag}}\right)}{2 \varepsilon_{\mathrm{PDMS}}+\varepsilon_{\mathrm{Ag}}+\left(\varepsilon_{\mathrm{PDMS}}-\varepsilon_{\mathrm{Ag}}\right)}\right],
$$

где $\varepsilon_{\text {PDMS }}$ - диэлектрическая проницаемость полидиметилсилоксана, а $\varepsilon_{\mathrm{Ag}}-$ серебра. С помощью этой формулы были рассчитаны спектры вещественной и мнимой частей эффективной диэлектрической проницаемости, приведенные на рис. 1. Данные для серебра и полидиметилсилоксана взяты из экспериментальных исследований [16].

Для создания оптоакустического генератора с широкими возможностями применения необходимо, чтобы его активная среда позволяла производить эффективное энергопреобразование в разных режимах ультразвукового исследования: как импульсами с большой скважностью, так и в режиме непрерывной модулированной волны (например, $\mathrm{CW}$-доплерография, которая активно внедряется для контроля показателей кровотока нестабильных пациентов $[17,18])$. Не менее чем эффективный нагрев системы важна ее адекватная температурная релаксация. Для оценки времени остывания гибридного металлоорганического материала было проведено моделирование в среде Comsol Multiphysics.

Для наночастиц радиусом $50 \mathrm{~nm}$, интегрированных в слой полидиметилсилоксана с объемными долями 17 и 29\%, было проведено моделирование остывания структуры на воздухе. В обоих расчетах количество наночастиц равнялось 1000. Моделировались остывание и перераспределение температуры в структуре исходя из начального предположения, что металлические наночастицы нагреты до $405 \mathrm{~K}$ (максимальный нагрев, не являющийся критическим для полидиметилсилоксана), а полидиметилсилоксан и окружающая среда изначально комнатной температуры $(293.15 \mathrm{~K})$. Целесообразно настраивать диапазон рабочих температур прибора ближе к границе, определяемой органическим компонентом гибридного материала, так как больший контраст с комнатной температурой обеспечит более эффективное охлаждение. На рис. 2, $a, b$ изображено распределение температуры в структуре с объемной долей наночастиц $29 \%$ через 40 и 60 ns. На рис. 2, с представлены зависимости максимальной температуры внутри структуры от времени. Видно, что за время порядка $100 \mathrm{~ns}$ структура с объемной долей наночастиц, составляющей 17\%, успевает релаксировать значительно лучше, чем структура с долей $29 \%$ (до $320 \mathrm{~K}$ против $350 \mathrm{~K}$ ).

В среде Comsol Multiphysics было проведено моделирование прохождения потока тепла через слой гибридного материала и таким образом установлены значения теплопроводности для разных объемных долей наночастиц серебра. Значения основных эффективных оптических и термодинамических характеристик гибридного материала приведены в таблице. На рис. 3 представлены зависимости температуропроводности и мнимой части показателя преломления от процентного содержания серебра. Обе величины растут с увеличением содер- 

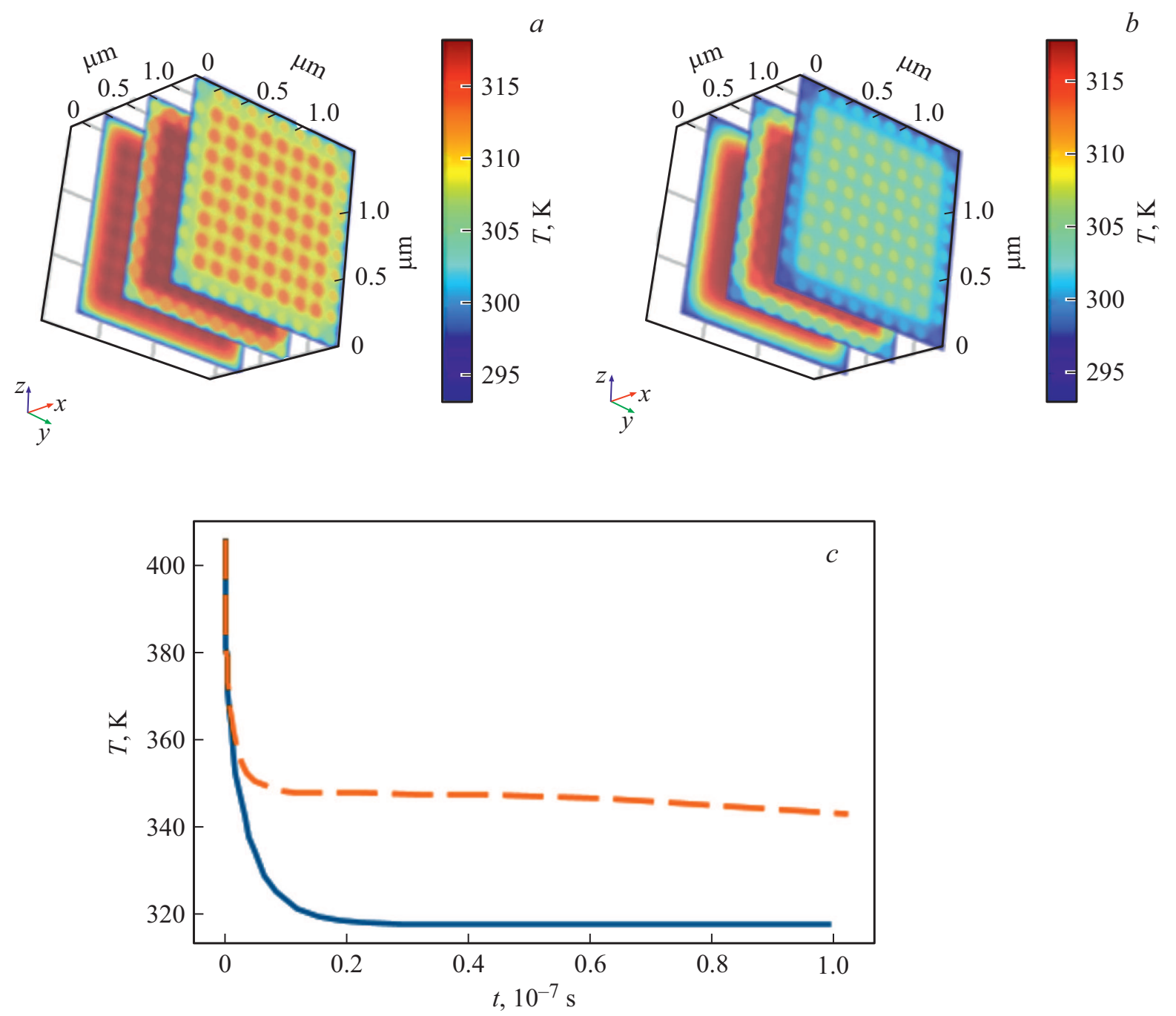

Рис. 2. Распределение температуры в структуре с объемной долей наночастиц, равной $29 \%$, через $40(a)$ и $60 \mathrm{~ns}(b)$. $c$ - графики падения температуры со временем при остывании материалов с объемными долями частиц 17\% (сплошная линия) и $29 \%$ (штриховая линия).

Зависимость теплопроводности $A$, температуропроводности $D$, теплоемкости $c$, плотности $\rho$, вещественной $n$ и мнимой $k$ частей показателя преломления от процентного содержания наночастиц серебра $f$

\begin{tabular}{|c|c|c|c|c|c|c|}
\hline$f, \%$ & $\begin{array}{c}A, \\
\mathrm{~W} /(\mathrm{m} \cdot \mathrm{K})\end{array}$ & $\begin{array}{c}D, \\
10^{-7} \mathrm{~m}^{2} / \mathrm{s}\end{array}$ & $\begin{array}{c}c, \\
\mathrm{~J} /(\mathrm{kg} \cdot \mathrm{K})\end{array}$ & $\begin{array}{c}\rho, \\
\mathrm{kg} / \mathrm{m}^{3}\end{array}$ & $\begin{array}{c}n \\
(\lambda=980 \mathrm{~nm})\end{array}$ & $\begin{array}{c}k \\
(\lambda=980 \mathrm{~nm})\end{array}$ \\
\hline 10 & 0.26 & 1.7 & 790 & 1917.5 & 0.024 & 21 \\
\hline 12.5 & 0.285 & 1.8 & 715 & 2155.63 & 0.027 & 25.3 \\
\hline 15 & 0.332 & 2.18 & 655 & 2393.75 & 0.028 & 26.1 \\
\hline 19 & 0.4 & 2.48 & 580.4 & 2774.75 & 0.031 & 29.8 \\
\hline 29 & 0.55 & 3.1 & 365 & 3727.25 & 0.038 & 37.2 \\
\hline $100(\mathrm{Ag})$ & 430 & 961 & 235 & 10490 & 0.04 & 6.9624 \\
\hline $\begin{array}{c}0 \\
\text { (PDMS) }\end{array}$ & 0.16 & 1.1 & 1460 & 965 & 1.3951 & 6.9624 \\
\hline
\end{tabular}

жания серебряных наночастиц, из чего можно сделать вывод, что в качестве активной среды оптоакустического преобразователя лучше использовать материал с долей наночастиц, близкой к 30\% (порог перколяции), но необ- ходимо также учитывать, что система должна успевать релаксировать на предполагаемых рабочих частотах.

Таким образом, показано, что гибридный материал, состоящий из полидиметилсилоксановой матрицы и ин- 


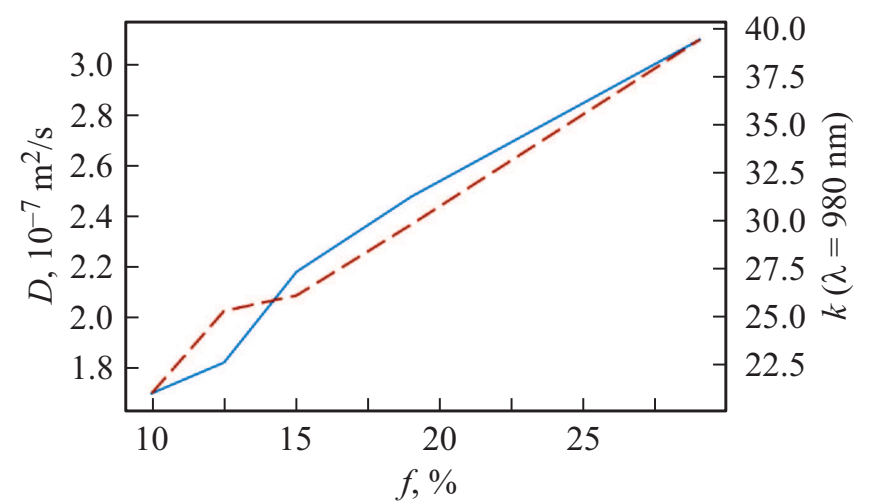

Рис. 3. Зависимость эффективной температуропроводности $D$ (сплошная линия) и мнимой части коэффициента преломления $k$ на длине волны $980 \mathrm{~nm}$ (штриховая линия) от объемной доли наночастиц серебра.

тегрированных в нее металлических наночастиц, может рассматриваться в качестве активной среды для оптоакустического генератора, так как поглощает на длине волны полупроводникового лазера и может обеспечивать достаточную температурную релаксацию для работы прибора на частотах порядка $10 \mathrm{MHz}$, рассчитаны эффективные оптические и термодинамические характеристики материала, которые могут быть использованы для проектирования прибора.

\section{Финансирование работы}

Работа поддержана Российским научным фондом (грант 21-12-00304).

\section{Конфликт интересов}

Авторы заявляют, что у них нет конфликта интересов.

\section{Список литературы}

[1] Y. Hou, J.-S. Kim, S.-W. Huang, S. Ashkenazi, L.J. Guo, M. O'Donnell, IEEE Trans. Ultrason. Ferroelectr. Freq. Control., 55 (8), 1867 (2008). DOI: 10.1109/TUFFC.2008.870

[2] X. Zou, N. Wu, Y. Tian, X. Wang, Opt. Express, 22 (15), 18119 (2014). DOI: 10.1364/OE.22.018119

[3] Л.М. Лямшев, УФН, $135 \quad$ (12), 637 (1981). DOI: $10.3367 /$ UFNr.0135.198112c.0637 [L.M. Lyamshev, Sov. Phys. Usp., 24 (12), 977 (1981). DOI: 10.1070/PU1981v024n12ABEH004757].

[4] V.P. Zharov, V.S. Letokhov, E.A. Ryabov, Appl. Phys., 12 (1), 15 (1977). DOI: 10.1007/BF00900062

[5] E.I. Girshova, A.P. Mikitchuk, A.V. Belonovski, K.M. Morozov, K.A. Ivanov, G. Pozina, K.V. Kozadaev, A.Yu. Egorov, M.A. Kaliteevski, Opt. Express, 28 (18), 26161 (2020). DOI: 10.1364/OE.400639

[6] M. Lal, M. Joshi, D.N. Kumar, C.S. Friend, J. Winiarz, T. Asefa, P.N. Prasad, MRS Proc., 519, 217 (1998).

DOI: $10.1557 /$ PROC-519-217
[7] C. Sanchez, B. Lebeau, MRS Bull., 26 (5), 377 (2001). DOI: $10.1557 / \mathrm{mrs} 2001.91$

[8] M.C. Orilall, U. Wiesner, Chem. Soc. Rev., 40, 520 (2011). DOI: $10.1039 / \mathrm{c} 0 \mathrm{cs} 00034 \mathrm{e}$

[9] M.A. Kaliteevski, S. Brand, J. Garvie-Cook, R.A. Abram, J.M. Chamberlain, Opt. Express, 16 (10), 7330 (2008). DOI: $10.1364 /$ OE. 16.007330

[10] A.J. Gallant, M.A. Kaliteevski, S. Brand, D. Wood, M. Petty, R.A. Abram, J.M. Chamberlain, J. Appl. Phys., 102 (2), 023102 (2007). DOI: 10.1063/1.2756072

[11] Y. Hou, S. Ashkenazi, S. Huang, M. O'Donnell, IEEE Trans. Ultrason. Ferroelectr. Freq. Control, 55 (12), 2719 (2008). DOI: 10.1109/TUFFC.2008.988

[12] J. Li, X. Lan, S. Lei, J. Ou-Yang, X. Yang, B. Zhu, Carbon, 145, 112 (2019). DOI: 10.1016/j.carbon.2019.01.025

[13] S.H. Lee, Y. Lee, J.J. Yoh, Appl. Phys. Lett., 106 (8), 081911 (2015). DOI: 10.1063/1.4913970

[14] Y. Li, Z. Guo, G. Li, S.-L. Chen, Opt. Express, 26 (17), 21700 (2018). DOI: 10.1364/OE.26.021700

[15] H. Won Baac, J.G. Ok, H.J. Park, T. Ling, S.-L. Chen, A.J. Hart, L.J. Guo, Appl. Phys. Lett., 97 (23), 234104 (2010). DOI: $10.1063 / 1.3522833$

[16] S. Babar, J.H. Weaver, Appl. Opt., 54 (3), 477 (2015). DOI: $10.1364 / A O .54 .000477$

[17] J.-É.S. Kenny, C.E. Munding, J.K. Eibl, A.M. Eibl, B.F. Long, A. Boyes, J. Yin, P. Verrecchia, M. Parrotta, R. Gatzke, P.A. Magnin, P.N. Burns, F.S. Foster, C.E.M. Demore, Sci. Rep., 11, 7780 (2021). DOI: 10.1038/s41598-021-87116-y

[18] B. Pialot, J. Gachelin, M. Tanter, J. Provost, O. Couture, IEEE Trans. Ultrason. Ferroelectr. Freq. Control, 67 (7), 1293 (2020). DOI: 10.1109/TUFFC.2020.2969080 